\title{
In Vivo Externalization of Phosphatidylserine and Phosphatidylethanolamine in the Membrane Bilayer and Hypercoagulability by the Lipid Peroxidation of Erythrocytes in Rats
}

\author{
Sushil K. Jain \\ Department of Pediatrics, Louisiana State University School of Medicine, Shreveport, Louisiana 71130
}

\begin{abstract}
Phospholipid distribution across erythrocyte membrane bilayer is asymmetrical. In normal erythrocytes, entire phosphatidylserine (PS) and most of the phosphatidylethanolamine (PE) is present on the cytoplasmic side of membrane bilayer, whereas phosphatidylcholine (PC) and sphingomyelin (SM) are predominantly present at the outer side of membrane bilayer. The present study was undertaken to determine whether membrane lipid peroxidation has any effect on the distribution of PS, PE, and $\mathrm{PC}$ across erythrocyte membrane bilayer in vivo in an animal model. Erythrocyte membrane lipid peroxidation was induced in rats by administering phenylhydrazine, an oxidant drug. Membrane phospholipid organization was determined by using bee venom phospholipase- $\mathrm{A}_{2}$ and indirectly by measuring clotting time on recalcification of normal human platelet-poor plasma in the presence of Russell's viper venom. Phenylhydrazine administration to rats caused significant membrane lipid peroxidation as measured by the accumulation of malonyldialdehyde (MDA), an end product of fatty acid peroxidation, as well as externalization of a significant portion of PS and PE from the inner to the outer side of membrane bilayer in erythrocytes. There was a significant positive correlation ( $r)$ between the amount of MDA accumulated in the erythrocytes and the movement of PS $(r=0.92)$ and PE $(r=0.96)$ from inner to the outer membrane bilayer and PC $(r=0.81)$ from outer to the inner membrane bilayer. Erythrocytes of phenylhydrazine-treated rats also showed significantly reduced clotting time. This reduction in clotting time had a significant positive correlation with MDA accumulation $(r=0.92)$ and PS externalization $(r=0.90)$. Both the effect of phenylhydrazine on erythrocyte membrane lipid peroxidation and alterations in phospholipid organization and coagulability were blocked when rats were simultaneously administered with vitamin $\mathbf{E}$ or $\mathbf{C}$ antioxidants.
\end{abstract}

\section{Introduction}

It is now well established that phospholipid distribution across erythrocyte membrane bilayer is asymmetrical (1). Sphingomyelin (SM) ${ }^{1}$ and phosphatidylcholine (PC) are predominantly

This work was presented at the 25th meeting of The American Society of Hematology, in San Francisco, 1983, and is published as an abstract (1983. Blood. 62:32).

Received for publication 17 April 1984 and in revised form 25 February 1985.

1. Abbreviations used in this paper: MDA, malonyldialdehyde; PC, phosphatidylcholine; PE, phosphatidylethanolamine; PS, phosphati-

J. Clin. Invest.

(C) The American Society for Clinical Investigation, Inc. 0021-9738/85/07/0281/06 \$1.00

Volume 76, July 1985, 281-286 present in the outer bilayer, and entire phosphatidylserine (PS) and most of phosphatidylethanolamine (PE) are present on the inner side of the membrane bilayer.

The presence of PS and PE on the inner side of erythrocyte membrane has biological significance. PS plays a very important role as a rate-enhancing co-factor in the blood coagulation cascade (2-5). Thus, the presence of procoagulant phospholipid on the outer surface of blood cells could result in a permanent condition of hypercoagulability. Chiu et al. (6) and Lubin et al. (7) have demonstrated that sickled cell membranes contain a significant proportion of PS and more PE in the outer bilayer. On the other hand, recent studies have reported increased amounts of malonyldialdehyde (MDA), an end product of lipid peroxidation, and a new lipid consisting of PS, PE, and MDA in untreated sickled cells $(6,8,9)$, providing evidence for in vivo peroxidative lipid damage to erythrocyte membrane in sickle cell disease. Hebbel et al. (10) also have shown that sickle cells can spontaneously generate superoxide radicals. The present study was undertaken to examine the possible relationship among erythrocyte membrane lipid peroxidative damage, altered phospholipid organization, and hypercoagulability in vivo.

\section{Methods}

Phenylhydrazine (Sigma Chemical Co., St. Louis, MO), an oxidant drug known to generate superoxide radicals $(11,12)$ and to initiate peroxidation of membrane lipids (12-14), was used to induce in vivo lipid peroxidation in the erythrocytes of rats. Sprague-Dawley rats $(150 \pm 15 \mathrm{~g})$ were intramuscularly administered with various doses $(0-30 \mathrm{mg} / \mathrm{kg}$ body weight) of phenylhydrazine- $0.15 \mathrm{M}$ sodium chloride for $2 \mathrm{~d}$. Phenylhydrazine was administered at the same time on each of the $2 \mathrm{~d}$ (at 20 and $44 \mathrm{~h}$ before their being killed). Control rats were sham-treated with equal volumes of $0.15 \mathrm{M}$ sodium chloride. In certain experiments, rats were simultaneously administered with vitamin $\mathrm{E}$ ( $d$ $\alpha$-tocopherol acetate) or C (L-ascorbic acid). At the end of treatments, overnight fasted rats were killed by collecting blood via heart puncture into heparinized syringes under light ether-anesthesia. Blood was centrifuged at $1,500 \mathrm{rpm}$ in an IEC PR 6000 centrifuge at $4^{\circ} \mathrm{C}$. Plasma and buffy coat were discarded. Residual cells were washed three times with 10 times volume of cold $0.15 \mathrm{M}$ sodium chloride.

Phospholipase treatment. To determine phospholipid organization we suspended erythrocytes in glycylglycine buffer at pH 7.4 (15) containing $100 \mathrm{mM} \mathrm{KCl}, 50 \mathrm{mM} \mathrm{NaCl}, 0.25 \mathrm{mM} \mathrm{MgCl}_{2}, 44 \mathrm{mM}$ sucrose, $10 \mathrm{mM}$ glycylglycine, and $0.25 \mathrm{mM} \mathrm{CaCl}_{2}$. The red cells were then exposed for $3 \mathrm{~h}$ to nonpermeable bee venom phospholipase- $\mathrm{A}_{2}$ (Sigma Chemical Co.), which specifically hydrolyses PC, PS, and PE present in the outer membrane bilayer (15). The incubation medium contained $0.25 \mathrm{ml}$ of packed erythrocytes plus $5 \mathrm{ml}$ glycylglycine buffer plus $50 \mathrm{U}$ of phospholipase- $\mathrm{A}_{2}$. Incubations were done in Erlenmeyer flasks in a shaking water bath at $37^{\circ} \mathrm{C}$ for time periods given in figures or tables. Various incubations contained respective flasks of erythrocytes not treated with phospholipase as controls. At the end of incubation

dylserine; RVV, Russell's viper venom; SM, sphingomyelin; TLC, thin-layer chromatography. 
time, phospholipase- $A_{2}$ action was terminated by adding $5 \mathrm{mM}$ EDTA to the incubation medium. A portion of the first supernatant was used to determine the extent of hemolysis during phospholipase treatment. Erythrocytes were subsequently washed with cold $0.15 \mathrm{M}$ sodium chloride three times, as described above, to remove any lysed cell ghost and enzyme into the supernatant before lipid extraction.

Lipid extraction and analyses. Lipid extraction of washed phospholipase-treated erythrocytes was carried out using isopropanol and chloroform by the method of Rose and Oklander (16). Lipid extracts were dried with nitrogen and then redissolved in $50 \mu$ l of chloroform. Phospholipid classes in the lipid extracts of phospholipase-treated cells were separated by two-dimensional thin-layer chromatography (TLC) on silica gel $\mathrm{H}$ plates (Brinkmann Instruments, Westbury, NY) using solvent systems consisting of chloroform-methanol-acetic acid-water/ 50:25:8:4 (vol/vol) in the first direction and chloroform-methanolwater/5:10:1 (vol/vol) in the second direction. Visualization of various lipid spots on TLC plate and phospholipid-phosphorus determination were carried out as described earlier (17). The extent of phospholipid degradation by phospholipase was assessed by calculating decrease in the ratio of unhydrolyzed phospholipid to SM. Phospholipase used does not act on SM. Two-dimensional TLC used separates lysolipids from other lipids. Phospholipase treatment of erythrocytes for up to 3 h did not cause $>4 \%$ hemolysis.

Russell's viper venom (RVV) clotting time. RVV clotting time was determined as described by $\mathrm{Zwaal}$ et al. (4). $0.1 \mathrm{ml}$ of citrated human plasma (centrifuged $15 \mathrm{~min}, 5,000 \mathrm{~g}$ ) was incubated in a fibrometer at $37^{\circ} \mathrm{C}$ for $30 \mathrm{~s}$ with $10 \mu \mathrm{l}$ of nearly $12 \mathrm{ng} \mathrm{RVV}$, followed by an addition of $0.1 \mathrm{ml}$ of washed unhemolyzed packed erythrocytes ( $\sim 85 \%$ hematocrit and similar amount of phospholipid) and $0.1 \mathrm{ml}$ of $25 \mathrm{mM}$ calcium chloride. Clotting time of erythrocytes of untreated rats was considered $100 \mathrm{~s}$ and clotting time of erythrocytes of treated rats is expressed as a percent of the controls. In this assay system, clotting factor $\mathrm{X}$ is activated by RVV. Because the conversion of prothrombin to thrombin by prothrombinase is the last step in the coagulation cascade requiring phospholipid, this assay system is used to assess the exposure of procoagulant lipid PS in the erythrocyte membrane.

Measurement of lipid peroxidation. Membrane peroxidative damage in the erythrocyte was determined by the following methods:

(a) Thiobarbituric acid-reactivity: MDA, an end product of fatty acid peroxidation reactions, can react with thiobarbituric acid to form a colored complex that has maximum absorbance at $532 \mathrm{~nm}$. Thiobarbituric acid-reactivity of erythrocytes was determined by the method of Stocks and Dormandy (18).

(b) Lipid fluorescence: lipid fluorescence was measured in the lipid extracts of erythrocytes. For this purpose, $0.4 \mathrm{ml}$ of packed erythrocytes were suspended into $3 \mathrm{ml}$ of isopropanol and $2 \mathrm{ml}$ of chloroform as described by Rose and Oklander (16). Tubes were vortexed and allowed to stand at room temperature for $\sim 30 \mathrm{~min}$, at which time these tubes were again vortexed and then centrifuged at $2,000 \mathrm{rpm}$ for $15 \mathrm{~min}$ in a refrigerated centrifuge. The lipid extract, i.e., clear supernatant, from each tube was transferred into another tube. Fluorescence in the lipid extracts was measured using spectro fluorometer model 650-10 (PerkinElmer Corp., Eden Prairie, MN) at excitation wave length of $400 \mathrm{~nm}$ and emission wavelength of $455 \mathrm{~nm}$. These wave lengths are characteristic of lipid complex formed by the cross-linking of aldehyde groups of MDA with the amino groups of PS and PE $(12,19)$. Previous studies have shown a linear correlation between increase in the lipid extract fluorescence at these excitation and emission wavelengths and the amount of exogenous MDA treatment in vitro to erythrocytes (9, 19). A relative increase in the lipid fluorescence is an indirect index of peroxidation of membrane lipids and MDA accumulation. Lipid fluorescence values presented in results are only relative Perkin-Elmer units obtained by setting the spectrofluorometer at band pass of 10 $\mathrm{nm}$ each of excitation and emission.

(c) Peroxidative membrane damage also was assessed by determining the amount of heterologous phospholipid/MDA adduct separated by TLC of erythrocyte lipid extracts as described earlier (9). The quantitation of phospholipid/MDA adduct on TLC gives the amount of MDA cross-linked between PE and PS, whereas fluorescence in the lipid extracts measures total MDA cross-linking between PE and PE, PS and PS, and PE and PS, i.e., both homologous and heterologous MDA adducts.

\section{Results}

There was a dose-dependent decrease in the erythrocyte count and hematocrit of the blood of rats administered with phenylhydrazine in comparison to control rats. Erythrocyte counts ( $10^{6}$ per cubic millimeter) were $7.5 \pm 0.5,7.4 \pm 0.3,6.4 \pm 0.6$, $6.6 \pm 0.3,5.7 \pm 0.4,5.4 \pm 0.6$, and $5.3 \pm 0.4$, and hematocrits $(\%)$ were $43 \pm 2,41 \pm 2,37 \pm 4,37 \pm 3,34 \pm 2,33 \pm 2$, and $32 \pm 1$ (mean \pm SD of three rats each) in rats administered with 0,5 , $10,15,20,25$, and $30 \mathrm{mg}$ phenylhydrazine $/ \mathrm{kg}$ body weight, respectively.

Table I gives the effect of phenylhydrazine administration on the phospholipid composition, MDA levels, and lipid extract fluorescence of the erythrocytes of rats. Phenylhydrazine treatment caused dose-dependent increase in the peroxidative lipid damage in the erythrocytes as seen by increase in the

Table I. Phospholipid Composition, MDA Levels, and Lipid Extract Fluorescence of Erythrocytes from Control and Phenylhydrazine-treated Rats

\begin{tabular}{|c|c|c|c|c|c|c|c|c|}
\hline \multirow{2}{*}{$\begin{array}{l}\text { Phenylhydrazine } \\
\text { treatment (mg/kg } \\
\text { body weight) }\end{array}$} & \multicolumn{6}{|c|}{$\%$ of total PL } & \multirow{2}{*}{$\begin{array}{l}\text { MDA } \\
(\mathrm{nmol} / \mathrm{ml} \\
\mathrm{PCV})\end{array}$} & \multirow{2}{*}{$\begin{array}{l}\text { Lipid } \\
\text { fluorescence } \\
\text { (relative) }\end{array}$} \\
\hline & LPC & SM & PC & $\mathbf{P S}+\mathbf{P I}$ & adduct & PE & & \\
\hline \multicolumn{9}{|l|}{0} \\
\hline (Control) & $2.3 \pm 0.1$ & $8.7 \pm 0.8$ & $47.5 \pm 1.2$ & $15.4 \pm 0.3$ & 0 & $25.7 \pm 0.7$ & $2.4 \pm 0.3$ & $26 \pm 3$ \\
\hline 5 & $2.5 \pm 0.2$ & $8.7 \pm 0.6$ & $48.1 \pm 1.0$ & $14.9 \pm 0.5$ & 0 & $25.8 \pm 0.4$ & $2.9 \pm 0.3$ & $32 \pm 2^{*}$ \\
\hline 10 & $2.1 \pm 0.1$ & $7.3 \pm 0.3$ & $49.6 \pm 0.3$ & $15.6 \pm 0.5$ & $0.2 \pm 0.1$ & $25.1 \pm 0.2$ & $3.6 \pm 0.5^{*}$ & $47 \pm 3^{*}$ \\
\hline 15 & $2.9 \pm 0.3^{*}$ & $8.2 \pm 1.0$ & $47.5 \pm 1.5$ & $14.5 \pm 0.3$ & $0.5 \pm 0.2$ & $25.7 \pm 0.2$ & $4.2 \pm 0.6^{*}$ & $50 \pm 2 *$ \\
\hline 20 & $2.6 \pm 0.1^{*}$ & $8.2 \pm 0.8$ & $50.6 \pm 1.9$ & $13.4 \pm 0.7^{*}$ & $0.7 \pm 0.2$ & $24.2 \pm 0.4$ & $4.1 \pm 0.3^{*}$ & $53 \pm 1^{*}$ \\
\hline 25 & $2.8 \pm 0.2^{*}$ & $8.4 \pm 0.8$ & $49.5 \pm 0.6$ & $13.5 \pm 0.2^{*}$ & $1.3 \pm 0.4$ & $24.3 \pm 0.1$ & $4.6 \pm 0.4^{*}$ & $54 \pm 2 *$ \\
\hline 30 & $2.5 \pm 0.3$ & $7.9 \pm 0.9$ & $48.8 \pm 0.1$ & $13.4 \pm 0.3^{*}$ & $1.6 \pm 0.3$ & $25.2 \pm 0.9$ & $5.1 \pm 0.4^{*}$ & $55 \pm 2^{*}$ \\
\hline
\end{tabular}

Values are mean $\pm \mathrm{SE}$ of five rats each. MDA levels were calculated by the molar extinction coefficient of MDA-thiobarbituric acid complex at $532 \mathrm{~nm}=1.56 \times 10^{5} \mathrm{~cm} / \mathrm{mmol}$. LPC, lysophosphatidylcholine; SM, sphingomyelin; PI, phosphatidylinositol; PL, phospholipid. * Values are statistically significant $(P<0.05)$ in comparison to control. 
phospholipid/MDA adduct formed by the cross-linking of MDA with the PS and PE, increased fluorescence in the lipid extracts, and increased free MDA levels. In addition, phenylhydrazine-treated erythrocytes contained a reduced proportion of PS when compared to controls. The proportion of SM, PC, and PE was similar in erythrocytes of both control and phenylhydrazine-treated rats.

Fig. 1 shows the effect of various concentrations of phenylhydrazine on the extent of phospholipase hydrolysis of phospholipids in erythrocytes. Phospholipase treatment to erythrocytes from normal rats showed no hydrolysis of PS, with nearly $11 \% \mathrm{PE}$ and $60 \%$ PC hydrolyzed, suggesting that no PS is present on the outer side of membrane bilayer. In contrast, erythrocytes from phenylhydrazine-administered rats showed as much as $26 \%$ PS, $41 \%$ PE, but only $46 \%$ PC hydrolysis by phospholipase, suggesting that a portion of PS and PE has moved from the inner to the outer side of membrane bilayer and that a portion of PC has moved from the outer to the inner side. Phospholipase treatment caused $2-4 \%$ hemolysis in flasks containing erythrocytes from phenylhydrazine-administered rats in comparison to $1-2 \%$ in control rats. Phospholipase can act on the both sides of membrane bilayer of lysed cells. Therefore, all lipid analyses were done after treated erythrocytes had been washed to remove any lysed cell into the supernatant. Moreover, contamination of degraded lipids from $4 \%$ lysed cells may not make a significant contribution in the observed values of as much as $26 \%$ PS and 41\% PE degradation in phospholipase-treated erythrocyte lipid extracts.

There was a significant positive correlation ( $r$ ) between the movement of PS $(r=0.92)$ and PE $(r=0.96)$ from the inner to the outer side of membrane bilayer and the extent of MDA accumulation in red cells (Fig. 1). This externalization of PS and $\mathrm{PE}$ in erythrocyte of phenylhydrazine-administered rats was associated with the simultaneous translocation of PC ( $r$ $=0.81$ ) from outer to the inner side of membrane bilayer. This abnormal externalization of PS and PE and the internalization of PC may be due both to their rapid transbilayer movement and to permanent rearrangement of PS and PE (20). The present study found nearly $60 \%$ degradation of PC by phospholipase- $A_{2}$ in the erythrocyte membrane of control rats in contrast to $48 \%$ reported by Renooij et al. (21). However, treatment with both phospholipase- $\mathrm{A}_{2}$ and sphingomyelinase as well as the use of specific exchange proteins have shown presence of up to $63 \%$ of PC in the outer bilayer of rat erythrocyte membrane $(21,22)$. Greater hydrolysis of $P C$ in rat erythrocyte membrane obtained in the present study seems to be due to $3 \mathrm{~h}$ time of phospholipase- $\mathrm{A}_{2}$ treatment in

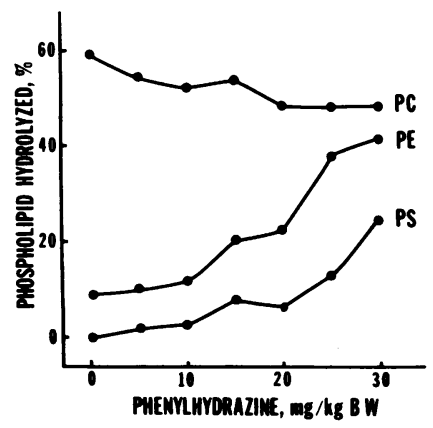

Figure 1. Phospholipid hydrolysis by bee venom phospholipase $A_{2}$ in erythrocytes of rats treated with various doses of phenylhydrazine. Values are means of five rats each. Variation between each value in the same group is $<10 \%$. SpragueDawley rats were administered intramuscularly with phenylhydrazine for $2 \mathrm{~d}$ at a daily dose as given in the figure. BW, body weight.

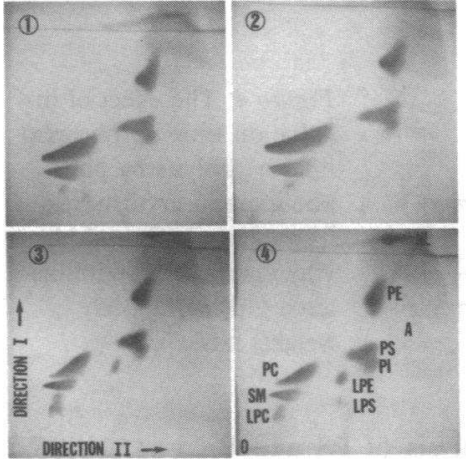

Figure 2. Thin-layer chromatography of erythrocytes lipid extracts. (1) erythrocytes from control rats; (2) erythrocytes from phenylhydrazine-administered rats; (3) phospholipasetreated erythrocytes of control rats; (4) phospholipasetreated erythrocytes of phenylhydrazine-administered $(20 \mathrm{mg} / \mathrm{kg}$ body weight) rats. TLC plates were developed in solvent systems (chloroform/methanol/acetic acid/water, 50:25:8:4 [vol/vol, first direction], and chloroform/methanol/water, 5:10:1 [vol/vol, second direction]). Iodine vapors were used to visualize phospholipids on the plate. Note formation of LPS and LPE by phospholipase action on PS and PE present in the erythrocyte outer membrane bilayer of phenylhydrazine-administered rats and the absence of LPS and very small LPE formed in the erythrocytes of phospholipasetreated control rats. LPC, lysophosphatidylcholine; LPE, lysophosphatidylethanolamine; phosphatidylinositol; NL, neutral lipids.

comparison to $2 \mathrm{~h}$ used by Renooij et al. (21). The extent of PE hydrolysis in the erythrocyte membrane of control rats is similar to the previous report (21).

Fig. 2 illustrates thin-layer chromatoplates of lipid extracts of phospholipase-treated erythrocytes from control and phenylhydrazine-administered rats. When phenylhydrazine-administered and control rats are compared, there is the appearance of lysophosphatidylserine and more lysophosphatidylethanolamine formation due to the degradation of outer bilayer PS and PE, respectively and less degradation of PC to lysophosphatidylcholine in the chromatoplates of phospholipase-treated erythrocytes of phenylhydrazine-administered rats. Migration of lysophosphatidylethanolamine and lysophosphatidylserine on TLC plates was confirmed by co-chromatography of these lipids with the erythrocyte lipid extracts as described previously (17).

Fig. 3 illustrates the association of MDA accumulation, PS externalization, and RVV clotting time in the erythrocytes of phenylhydrazine-treated rats. There was a significant correlation between reduction in the clotting time and the MDA accumulation $(r=0.92)$ and PS externalization $(r=0.90)$ in the erythrocytes.

To determine possible phospholipase leakage into the erythrocyte as a factor in initiating hydrolysis of PS and PE present in the inner membrane bilayer, we studied the time course of phospholipase action. The data presented in Fig. 4

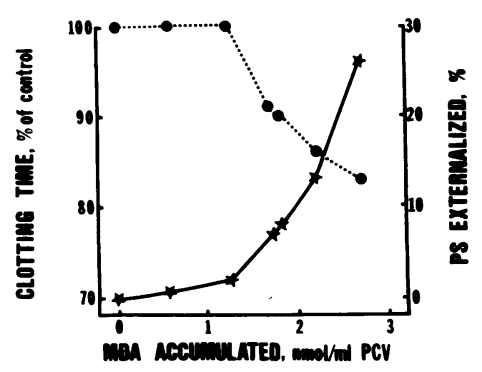

Figure 3. The relationship among malonyldialdehyde accumulation, PS externalization, and RVV clotting time of erythrocytes of phenylhydrazine-treated rats. Values are means of five rats each. Variation between each rat of the same group is $<10 \%$. $\bullet$, clotting time (percentage of control); *, PS externalized (\%). 


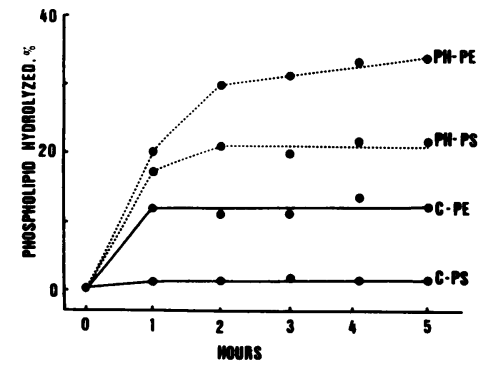

Figure 4. The effect of incubation time on phospholipid hydrolysis by phospholipase in erythrocytes from control $(C)$ and phenylhydrazine ( $\mathrm{PH})$-administered $(30 \mathrm{mg} / \mathrm{kg}$ body weight) rats.

show that maximum hydrolysis of PS and PE was achieved within $2 \mathrm{~h}$ of incubation. The extent of phospholipid hydrolysis remained the same even when erythrocytes were incubated with twofold quantities of phospholipase or when they were incubated for longer periods. This suggests that the membrane of erythrocytes from phenylhydrazine-administered rats is not permeable to the phospholipase protein molecule. Moreover, hydrolysis of the inner bilayer phospholipids of any erythrocyte would have caused lysis and its subsequent removal in the supernatant during washing of phospholipase-treated erythrocytes.

Phenylhydrazine administration is known to induce reticulocytosis in the circulation (23). To examine any contribution of reticulocytes in the observed changes in lipid peroxidation and phospholipid organization in erythrocytes from phenylhydrazine-administered rats, we studied another group of rats in which reticulocytosis was induced by bleeding. The data in Table II show that phospholipid hydrolysis by phospholipase was the same in erythrocytes from control and bled rats. Thus, the increased level of reticulocytes in the blood of phenylhydrazine-administered rats does not appear to contribute to the increased lipid peroxidation and altered phospholipid distribution observed in these cells.

Table III gives the effect of vitamins $E$ and $C$ supplementation on lipid peroxidation, phospholipid organization, and RVV clotting time of erythrocytes from control and phenylhydrazine-administered rats. Externalization of PS and PE, MDA accumulation, and reduction in RVV clotting time were blocked when rats were given these antioxidants. This suggests

Table II. Effect of Bleeding of Rats on the Phospholipase Hydrolysis of Erythrocyte Phospholipids

\begin{tabular}{|c|c|c|c|}
\hline & \multirow[b]{2}{*}{ Reticulocytosis } & \multicolumn{2}{|c|}{ Phospholipid hydrolysis } \\
\hline & & PS & PE \\
\hline & $\%$ & $\%$ of total & \% of total \\
\hline Control & $1.1 \pm 0.3^{*}$ & 0 & $11.7 \pm 0.8^{*}$ \\
\hline Bled & $18.5 \pm 1.6 \ddagger$ & 0 & $12.8 \pm 1.6^{*}$ \\
\hline $\begin{array}{l}\text { Phenylhydrazine } \\
\text { (15 mg/kg }\end{array}$ & & & \\
\hline body weight) & $17.4 \pm 2.1 \ddagger$ & $7.3 \pm 0.7$ & $27.8 \pm 1.5 \ddagger$ \\
\hline
\end{tabular}

Values are mean $\pm \mathrm{SE}$ of four control, four bled, and five phenylhydrazine-treated rats observations. Bleeding of rats was done by cutting tail end and letting nearly $2 \mathrm{ml}$ of blood three times at 12,24 , and $48 \mathrm{~h}$ before killing the rats. Tail was massaged with isopropanol swabs during bleeding for easy flow of blood.

* $\ddagger$ Differences between values marked * and $\ddagger$ were significant $(P<0.01)$. that externalization of PS and PE in erythrocytes of phenylhydrazine-administered rats is indeed associated with the peroxidative lipid damage.

\section{Discussion}

The occurrence of membrane lipid peroxidation and its role in the pathophysiology of erythrocytes has been examined by various investigators $(9,19,24-34)$. Previous studies have shown that erythrocyte lipid peroxidation can cause increased membrane permeability (30), increased hemoglobin binding to the membrane (31), reduced whole cell deformability (32, 33), cellular dehydration, and reduced erythrocyte survival (33). The present study has documented in vivo that lipid peroxidation can cause hypercoagulability and externalization of PS and PE in the membrane bilayer of erythrocytes. A previous in vitro study (34) also has shown destabilization of phospholipid organization in erythrocytes treated with peroxide or malonyldialdehyde.

In normal erythrocytes no PS and only small amounts of PE are present in the outer membrane bilayer. Zwaal et al. (4) have provided evidence that asymmetric distribution of membrane phospholipids in blood cells may serve a biological purpose by contributing to the delicate balance between regulating hemostasis and avoiding thrombosis. In addition, observations by Wilschut and Papahadjopoulos (35) that negatively charged PS and calcium ions are essential for cell to cell contact in cell fusion indicate that erythrocytes with abnormal externalization of PS may be susceptible to adherence to endothelial cells, causing microvascular occlusions. In at least one disease state, sickle cell disease, various investigators have documented in vivo peroxidative lipid damage $(8,9)$, externalization of PS and PE $(6,7)$, and, in vitro, greater adherence to endothelial cells $(36,37)$, activation of blood coagulation (38), and spontaneous generation of superoxide radicals (10) by sickle cells.

Phenylhydrazine can generate superoxide radicals in vitro $(11,12)$. Its administration to animals can induce peroxidative lipid damage of erythrocytes, resulting in the accumulation of MDA, an end product of fatty acid peroxidation reactions, and the cross-linking of MDA with aminophospholipids. This in turn can give rise to increased fluorescence in the lipid extracts $(12,14,19)$ and to a new lipid spot, which has been previously characterized as a cross-linked adduct of PS, PE, and MDA (9).

Decreased PS levels in the phenylhydrazine-administered rats may be due to the fact that PS is rich in unsaturated fatty acids, which are most vulnerable to peroxidative breakdown (39), and to the fact that PS, unlike other phospholipids, does not participate in the exchange process with plasma phospholipids (40) and, hence, cannot be renewed. PS is a charged phospholipid known to have a regulatory role in blood coagulation (2-5). Externalization of PS at the outer side of erythrocytes may act as a catalytic surface on which various coagulation factors can interact, resulting in a condition of hypercoagulability.

The mechanism responsible for the externalization of PS and $P E$ in the lipid bilayer of erythrocyte membrane is unknown. Oxidation of spectrin by sulfhydryl oxidizing agents has been shown to cause increased amounts of PE and PS that has been exposed to the outer leaflet of erythrocyte membrane bilayer $(15,41)$. Recent studies also have reported externaliza- 
Table III. Phospholipid Hydrolysis by Phospholipase- $A_{2}$ and RVV Clotting Time of Erythrocytes from Phenylhydrazine- and/or Antitoxidant-administered Rats

\begin{tabular}{|c|c|c|c|c|c|}
\hline & \multirow[b]{2}{*}{ Malonyldialdehyde } & \multicolumn{3}{|c|}{ Phospholipid hydrolysis } & \multirow{2}{*}{$\begin{array}{l}\text { RVV clotting } \\
\text { time }\end{array}$} \\
\hline & & PC & PE & PS & \\
\hline & $n m o l / m l ~ P C V$ & $\%$ & $\%$ & $\%$ & $\%$ of control \\
\hline Control & $2.2 \pm 0.3$ & $61.1 \pm 1.7$ & $11.9 \pm 1.0$ & 0 & $100 \pm 1$ \\
\hline \multicolumn{6}{|l|}{ Vitamin E $(2.5 \mathrm{mg} / \mathrm{kg}$ body } \\
\hline weight) & $2.4 \pm 0.4$ & $60.2 \pm 1.0$ & $9.2 \pm 0.7^{*}$ & 0 & $101 \pm 2$ \\
\hline Vitamin C (10 mg/kg body weight $)$ & $2.3 \pm 0.5$ & $60.9 \pm 0.6$ & $12.1 \pm 0.6$ & 0 & $102 \pm 2$ \\
\hline \multicolumn{6}{|l|}{ Phenylhydrazine $(20 \mathrm{mg} / \mathrm{kg}$ body } \\
\hline weight) & $4.2 \pm 0.3^{*}$ & $52.1 \pm 1.9^{*}$ & $28.9 \pm 1.3^{*}$ & $8.8 \pm 0.8^{*}$ & $90 \pm 1^{*}$ \\
\hline Vitamin $\mathrm{E}+$ phenylhydrazine & $3.1 \pm 0.4 \ddagger$ & $58.4 \pm 0.9 \ddagger$ & $15.4 \pm 1.7 \ddagger$ & $4.2 \pm 0.5 \ddagger$ & $94 \pm 2 \ddagger$ \\
\hline Vitamin $C+$ phenylhydrazine & $3.2 \pm 0.4 \S$ & $57.2 \pm 1.3 \S$ & $18.7 \pm 1.0 \S$ & $3.9 \pm 0.6 \S$ & $95 \pm 2 \S$ \\
\hline
\end{tabular}

Values are mean $\pm \mathrm{SE}$ of five observations. Differences in values marked ${ }^{*}$ in comparison to controls, between ${ }^{*}$ and $\ddagger$, and between ${ }^{*}$ and $\S$ in the same column are statistically significant $(P<0.05)$.

tion of PS and PE in calcium-loaded (42), hereditary spectrindeficient (43), and peroxide-treated (34) human erythrocytes. A previous study has shown that in vitro treatment of erythrocytes with MDA can cause destabilization of membrane phospholipids organization (34). Erythrocytes from phenylhydrazine-administered rats have a significant accumulation of MDA and can have a marked reduction in spectrin (14), which may be responsible for the destabilization of phospholipid organization observed in the present study in cells with in vivo peroxidative lipid damage. Erythrocytes from phenylhydrazineadministered rats also have elevated calcium levels (44). Elevated membrane-bound calcium can cause reduction in spectrin (45) and accentuate membrane lipid peroxidation (46), which, in turn, can contribute to externalization of PS and PE in erythrocytes from phenylhydrazine-administered rats. MDA formed during lipid peroxidation also can cross-link through Schiff's base formation between PS and PS, PE and PE, and PS and PE $(9,47)$. If this chemical cross-linking between two membrane lipids is not uniform across membrane bilayer, physical forces binding the lipid-lipid and/or lipid-protein may be disturbed, contributing to the destabilization of membrane phospholipid organization in erythrocytes with lipid peroxidation.

\section{Acknowledgments}

I am grateful to Mrs. Ethelyn B. Lahaye for her technical assistance, to Mrs. Kathy Jones for typing, and to Barbara McRoberts for editing this manuscript.

This study was supported in part by a grant-in-aid from the Hoffmann-La Roche, Inc., and by an Edward P. Stiles Award from the Louisiana State University Medical Center at Shreveport.

\section{References}

1. van Deenen, L. L. M. 1981. Topology and dynamics of phospholipids in membranes. FEBS (Fed. Eur. Biochem. Soc.) Lett. 123:3-15.

2. Zwaal, R. F. A. 1978. Membrane and lipid involvement in blood coagulation. Biochim. Biophys. Acta. 515:163-205.

3. Marcus, A. J. 1966. The role of lipid in blood coagulation. Adv. Lipid Res. 4:1-37.
4. Zwaal, R. F. A., P. Comfurius, and L. L. M. van Deenen. 1977. Membrane asymmetry and blood coagulation. Nature (Lond.). 268: 358-360.

5. Mannhalter, C., S. Schiffman, and E. Deutsch. 1984. Phospholipids accelerate factor IX activation by surface bound factor IXa. $\mathrm{Br}$. J. Haematol. 56:261-271.

6. Chiu, D., B. Lubin, and S. B. Shohet. 1979. Erythrocyte membrane lipid organization during the sickling process. Br. J. Haematol. 41:223-234.

7. Lubin, B., D. Chiu, J. Bastacky, B. Roelofson, and L. L. M. van Deenen. 1981. Abnormalities in membrane phospholipid organization in sickled erythrocytes. J. Clin. Invest. 67:1643-1649.

8. Das, S. K., and R. C. Nair. 1980. Superoxide dismutase, glutathione peroxidase, catalase and lipid peroxidation of normal and sickled erythrocytes. Br. J. Haematol. 44:87-92.

9. Jain, S. K., and S. B. Shohet. 1984. A novel phospholipid in irreversibly sickled cells: evidence for in vivo peroxidative membrane damage in sickle cell disease. Blood. 63:362-367.

10. Hebbel, R. P., J. W. Eaton, M. Balasingam, and M. H. Steinberg. 1982. Spontaneous oxygen radical generation by sickle erythrocytes. J. Clin. Invest. 70:1253-1259.

11. Goldberg, B., A. Stern, and J. Peisach. 1975. The mechanism of superoxide generation by the interaction of phenylhydrazine with hemoglobin. J. Biol. Chem. 250:2401-2403.

12. Jain, S. K., and P. Hochstein. 1979. Generation of superoxide radicals by hydrazine: its role in phenylhydrazine-induced hemolytic anemia. Biochim. Biophys. Acta. 586:128-136.

13. Jain, S. K., and D. Subrahmanyam. 1978. On the mechanism of phenylhydrazine-induced hemolytic anemia. Biochem. Biophys. Res. Commun. 82:1320-1324.

14. Jain, S. K., and P. Hochstein. 1980. Membrane alterations in phenylhydrazine-induced reticulocytes. Arch. Biochem. Biophys. 201: 683-687.

15. Haest, C. W. M., G. Plasa, D. Kamp, and B. Deuticke. 1978. Spectrin as a stabilizer of the phospholipid asymmetry in the human erythrocyte membrane. Biochim. Biophys. Acta. 509:21-32.

16. Rose, H. G., and M. Oklander. 1965. Improved procedure for the extraction of lipids from human erythrocyte. J. Lipid Res. 6:528531 .

17. Jain, S. K., and D. Subrahmanyam. 1978. Two-dimensional thin-layer chromatography of polar lipids. Ital. J. Biochem. 27:11-19.

18. Stocks, J., and T. L. Dormandy. 1971. The autoxidation of human red cell lipids induced by hydrogen peroxide. Br. J. Haematol. 20:95-111.

19. Jain, S. K., and P. Hochstein. 1980. Polymerization of membrane 
components in aging red blood cells. Biochem. Biophys. Res. Commun 92:247-254.

20. Franck, P. F. H., D. T. Y. Chiu, J. A. F. Opden Kamp, B. Lubin, L. L. M. Van Deenen, and B. Roelofson. 1983. Accelerated transbilayer movement of phosphatidylcholine in sickled erythrocytes. A reversible process. J. Biol. Chem. 258:8435-8442.

21. Renooij, W., M. G. Van Golde, R. F. A. Zwaal, and L. L. M. Van Deenen. 1976. Topology asymmetry of phospholipid metabolism in rat erythrocyte membranes. Evidence for flip-flop of lecithin. Eur. J. Biochem. 61:53-58.

22. Crain, R. C., and D. B. Zilversmit. 1980. Two nonspecific phospholipid exchange proteins from beef liver. 2. Use in studying the asymmetry and transbilayer movement of phosphatidylcholine, phosphatidylethanolamine, and sphingomyelin in intact rat erythrocytes. Biochemistry. 19:1440-1447.

23. Jain, S. K., and D. Subrahmanyam. 1976. Studies on lipids of red blood cells in phenylhydrazine-treated albino rats. Indian $J$. Biochem. Biophys. 13:155-157.

24. Carrell, R. W., C. C. Winterbourn, and E. A. Rachmilewitz. 1975. Activated oxygen and haemolysis. Br. J. Haematol. 30:259-264.

25. Dodge, J. T., G. Cohen, H. J. Kayden, and G. B. Phillips. 1967. Peroxide hemolysis of red blood cells from patients with abetalipoproteinemia (acanthocytosis). J. Clin. Invest. 46:357-368.

26. Harm, W., N. L. Fortier, H. U. Lutz, G. Fairbanks, and L. Snyder. 1979. Increased erythrocyte lipid peroxidation in hereditary xerocytosis. Clin. Chim. Acta. 99:121-128.

27. Rachmilewitz, E. A., B. H. Lubin, and S. B. Shohet. 1976. Lipid membrane peroxidation in beta thalassemia major. Blood. 47: 495-505.

28. Flynn, T. P., G. J. Johnson, and D. W. Allen. 1981. Oxidative damage of erythrocyte membrane lipids and proteins in hemoglobin Koln disease. Clin. Res. 29:718A. (Abstr.)

29. Jacob, H. S., and S. E. Lux. 1968. Degradation of membrane phospholipids and thiols in peroxide hemolysis: studies in vitamin E deficiency. Blood. 32:549-568.

30. Chiu, D., B. Lubin, and S. B. Shohet. 1982. Peroxidative reactions in red cell biology. In Free Radicals in Biology. W. Pryor, editor. Academic Press, Inc., New York. 5:115-160.

31. Sauberman, N., J. Piotrowski, W. Joshi, N. Fortier, and L. M. Snyder. 1981. Irreversible spectrin-hemoglobin crosslinkages associated with oxidant hypersensitivity in pathologic or artificially dehydrated red cells. Blood. 58:34A. (Abstr.)

32. Pfafferott, C., H. J. Meiselman, and P. Hochstein. 1982. The effect of malonyldialdehyde on erythrocyte deformability. Blood. 59: 12-15.

33. Jain, S. K., N. Mohandas, M. R. Clark, and S. B. Shohet. 1983. The effect of malonyldialdehyde, a product of lipid peroxidation, on the deformability, dehydration and ${ }^{51} \mathrm{Cr}$-survival of erythrocytes. Br. J. Haematol. 53:247-255.

34. Jain, S. K. 1984. The accumulation of malonyldialdehyde, a product of fatty acid peroxidation, can disturb aminophospholipid organization in the membrane bilayer of human erythrocytes. J. Biol. Chem. 259:3391-3394.

35. Wilschut, J., and D. Papahadjopoulos. 1979. Ca-induced fusion of phospholipid vesicles monitored by mixing of aqueous contents. Nature (Lond.). 281:690-693.

36. Hoover, R., R. Rubin, G. Wise, and R. Warren. 1979. Adhesion of normal and sickle erythrocytes to endothelial monolayers cultures. Blood. 54:872-876.

37. Hebbel, R. P., O. Yamada, C. F. Moldow, M. S. Jacob, J. G. Whitè, and J. W. Eaton. 1980. Abnormal adherence of sickle erythrocytes to cultured vascular endothelium. Possible mechanism for microvascular occlusion in sickle cell disease. J. Clin. Invest. 65:154160.

38. Chiu, D., B. Lubin, B. Roelofson, and L. L. M. van Deenen. 1981. Sickled erythrocytes accelerate clotting in vitro: an effect of abnormal membrane asymmetry. Blood. 58:398-401.

39. Dodge, J. T., and G. B. Phillips. 1967 Composition of phospholipids and of phospholipid fatty acids and aldehydes in human red cells. J. Lipid Res. 8:667-675.

40. van Deenen, L. L. M., and J. deGier. 1974. Lipids of the red cell membrane. In The Red Cell. D. M. Surgenor, editor. Academic Press, Inc., New York. 1:147-211.

41. Haest, C. W. M., and B. Deuticke. 1976. Possible relationship between membrane proteins and phospholipid asymmetry in the human erythrocyte membrane. Biochim. Biophys. Acta. 436:353-365.

42. Chiu, D., R. Schwartz, M. Yee, and B. Lubin. 1983. Calcium induced abnormal membrane phospholipid organization in human erythrocytes. Blood. 62:30A. (Abstr.)

43. Lubin, B., D. Chiu, R. S. Schwartz, B. Cooper, K. John, L. Wolf, and S. E. Lux. 1983. Abnormal membrane phospholipid organization in spectrin deficient human red cells. Blood. 62:34A. (Abstr.)

44. Shalev, O., M. N. Leida, R. P. Hebbel, M. S. Jacob, and J. W. Eaton. 1981. Abnormal erythrocyte calcium hemostasis in oxidantinduced hemolytic disease. Blood. 58:1232-1235.

45. Lorand, L., G. E. Siefring, and L. Lowe-Krentz. 1979. Enzymatic basis of membrane stiffening in human erythrocytes. Semin. Hematol. 16:65-74.

46. Jain, S. K., and S. B. Shohet. 1981. Calcium potentiates the peroxidation of erythrocyte membrane lipids. Biochim. Biophys. Acta. 642:46-54

47. Bidlack, W. R., and A. L. Tappel. 1973. Fluorescence products of phospholipids during lipid peroxidation. Lipids. 8:203-207. 